\title{
Sulfur deposition in sour gas reservoirs: laboratory and simulation study
}

\author{
Guo Xiao*, Du Zhimin, Yang Xuefeng, Zhang Yong and Fu Dekui \\ State Key Laboratory of Oil and Gas Reservoir Geology and Exploitation, Southwest Petroleum University, \\ Chengdu, Sichuan 610500, China
}

\begin{abstract}
Sulfur deposition in the formation, induced by a reduction in the solubility of the sulfur in the gas phase, may significantly reduce the inflow performance of sour gas wells and some wells in sour gas reservoirs have even become completely plugged with deposited sulfur within several months. Accurate prediction and effective management of sulfur deposition are crucial to the economic viability of sour gas reservoirs.

In this paper, a dynamic flow experiment was carried out to investigate formation damage resulting from sulfur deposition using an improved experimental method. The core sample was extracted from the producing interval of the LG2 well, LG gas field in the Sichuan Basin. The experimental temperature was $26{ }^{\circ} \mathrm{C}$ and the initial pressure was $19 \mathrm{MPa}$. The displacement pressure continuously decreased from 19 to $10 \mathrm{MPa}$, and the depletion process lasted 15 days. Then the core was removed and dried. The core mass and core permeability were measured before and after experiments. Experimental results indicated that the core mass increased from $48.372 \mathrm{~g}$ before experiment to $48.386 \mathrm{~g}$ afterwards, while the core permeability reduced from 0.726 to 0.608 md during the experiment. Then the core was analyzed with a scanning electron microscope (SEM) and energy-dispersive X-ray mapping. The deposition pattern and micro-distribution of elemental sulfur was observed and the deposited elemental sulfur distributed as a film around the pore surface.

In addition, a preliminary three-dimensional, multi-component model was developed to evaluate the effect of sulfur deposition on production performance, and the effect of production rate on sulfur deposition was also investigated. Simulation results indicated that the stable production time would be shortened and the gas production rate would be decreased once sulfur deposited in the formation. The increase in deposited sulfur at high flow rates may be attributed to a bigger pressure drop than that at low gas flow rates. Gas production rate has a severe effect on sulfur saturation in the grid of producing well located in sour gas reservoir. The work suggests sulfur deposition should be considered to correctly predict production performance and gas production rate should be optimized in order to control or retard sulfur deposition during the development of sour gas reservoir.
\end{abstract}

Key words: Sour gas reservoir, sulfur deposition, experiment, numerical simulation, formation damage

\section{Introduction}

Elemental sulfur is often present in appreciable quantities in sour gas under reservoir conditions (Brunner and Woll, 1980; Brunner et al, 1988). Reduction in pressure and temperature generally reduces the solubility of sulfur in sour gas. Once the reservoir fluid has reached a sulfur-saturated state, further reduction in pressure and temperature will cause sulfur to deposit. On the other hand, the sulfur in the gas phase also reacts to form a hydrogen polysulfide species (Hyne and Derdall, 1980; Hyne, 1983). Since high pressure and temperature favor polysulfide formation, deposition of elemental sulfur occurs when changes in pressure and temperature alter the decomposition of polysulfide to

*Corresponding author. email: guoxiao@swpu.edu.cn Received May 6, 2009 elemental sulfur and $\mathrm{H}_{2} \mathrm{~S}$.

Many studies were focused on sulfur production and/ or deposition in gas/oil wellbore holes, especially of gas reservoirs (Brunner and Woll, 1980; Hyne, 1968; Roberts, 1997). The problem of elemental sulfur deposition has been mainly covered in the areas of chemical engineering, gas processing, and chemical analysis (Sung and Johnson, 1989; Flowers, 1990; Aitani, 1993). The proposed treatments were chemical separation (Beskov et al, 1989) or biological and microbial treatments (Gasiorek, 1994; Ruitenberg et al, 1999). On the other hand, limited research has been devoted to sulfur precipitation in gas/oil reservoir rocks. Kuo and Colsmann (1966) developed the first mathematical model of a solid phase precipitation in porous media and its influence on fluid flow. The model considered elemental sulfur as some of the dissolved sulfur precipitates from the solution as a result of depletion of reservoir pressure. The results 
of the study showed a rapid buildup of solid sulfur around the well and significant deposition near the outer boundary of the reservoir. Roberts (1997) have used a conventional black-oil reservoir simulator to model sulfur depositional processes and described significant flow impairment induced by sulfur deposition for a history match of the Waterton field case. Lately, Du et al (2006) have presented a new gasliquid-solid coupling model for fractured carbonate gas reservoirs with a high $\mathrm{H}_{2} \mathrm{~S}$-content, accounting for sulfur deposition, phase behavior variation, geochemical rockwater-gas interactions and adsorption. They compared the run results with the Roberts' calculation results in the literature (Roberts, 1997) and analyzed the reason for the differences of the development indexes between these two models. Hyne (1968) presented a survey of more than 100 producing wells in Canada and Europe about field operations of sour gas production. The survey focused on sulfur deposition at the bottom of producing wells and showed that high bottom hole and wellhead temperature and low wellhead pressure provide favorable conditions for sulfur deposition in well tubing. Al-Awadhy et al (1998) performed the first study to investigate sulfur deposition in carbonate oil reservoirs. They conducted a single experiment and developed a numerical model describing the phenomena. Abou-Kassem (2000) studied numerically and experimentally the deposition of elemental sulfur in porous media using gas and oil flow systems. The results indicated the existence of permeability damage due to elemental sulfur deposition. Shedid and Zekri (2002) conducted a detailed experimental study using a wide range of applied flow rates, different initial concentrations of sulfur, and different rock permeability values. The results of the study stressed the severity of the problem associated with sulfur deposition for different flow rates and under different initial sulfur concentrations of the crude oil. Shedid and Zekri (2004) carried out ten dynamic flow experiments under different flow rates, using different crude oils of different sulfur and asphaltene concentrations, to investigate the simultaneous deposition of sulfur and asphaltene in porous media. Experimental results indicated that the increase in simultaneous sulfur and asphaltene concentrations in the flowing oil could increase and accelerate the permeability damage in carbonate reservoirs.

In this paper, a dynamic flow experiment was carried out to investigate formation damage resulting from sulfur deposition using an improved experimental method. In addition, a preliminary three-dimensional, multi-component model was developed to evaluate the influences of sulfur deposition on production performance. The effect of production rate on sulfur deposition was also investigated.

\section{Experimental investigation of sulfur deposition}

Up to now, many experiments for modeling sulfur deposition in cores from oil reservoirs have been conducted, while few experiments have been made for high sulfur gas reservoirs globally. There are four main reasons for limited experiments of high sulfur gas reservoirs. (1) High risk for safety. In the experiment process, safety must be ensured because $\mathrm{H}_{2} \mathrm{~S}$ is hypertoxic. (2) Few experimental methods for reference. Reduction in pressure and temperature generally reduces the solubility of sulfur in sour gas. Once the reservoir fluid has reached a sulfur-saturated state, further reduction in pressure and temperature will cause sulfur to deposit. The requirement for experiment equipment of high sulfur gas reservoirs is much stricter than for liquid experiment equipment under the same conditions. (3) Longer experimental period. Variation of temperature and pressure can result in elemental sulfur deposition. However, for the special reservoirs, it maybe take a long time to make it happen. (4) Experimental results are uncertain. Because of the limit of research period, the core experiment can not infinitely extend time, which could make the results of experiment different from actual state. For these four reasons, it is very difficult to evaluate the elemental sulfur deposited in the core from high sulfur gas reservoirs. Therefore, as there were no ready-made experimental methods used for reference conditions, we have independently designed an experimental process and assembled relevant experimental components. Aiming at these key technological difficulties during gas production in high temperature high pressure high $\mathrm{H}_{2} \mathrm{~S}-\mathrm{CO}_{2}$ gas reservoirs, on the basis of improved experimental testing method and process, formation damage resulting from sulfur deposition has been conducted by using experimental and numerical simulation methods.

\subsection{Deposited sulfur in core samples}

An experimental set up consists of a core holder, a measuring pump, a sample preparation, a container, a corrosion proof pressure gauge, a voltage regulator, a confining pressure pump, a back pressure valve, a back pressure pump, a gas flow meter, and a ventilated fume hood, as shown in Fig. 1.

The gas samples from TD5-1 well were flooded through the actual cores to test the elemental sulfur deposition. The composition of well head gas sample of X gas reservoir is listed in Table 1. The scanning electron microscope (SEM) was used to evaluate the sulfur deposited along the actual cores.

Table $1 \mathrm{X}$ gas reservoir fluid composition

\begin{tabular}{cc}
\hline Component & $\begin{array}{c}\text { Mole fraction } \\
\text { mol\% }\end{array}$ \\
\hline $\mathrm{N}_{2}$ & 0.5000 \\
$\mathrm{H}_{2} \mathrm{~S}$ & 6.8600 \\
$\mathrm{CO}_{2}$ & 2.7600 \\
$\mathrm{C}_{1}$ & 89.6300 \\
$\mathrm{C}_{2}$ & 0.2100 \\
$\mathrm{C}_{3+}$ & 0.0200 \\
\hline
\end{tabular}




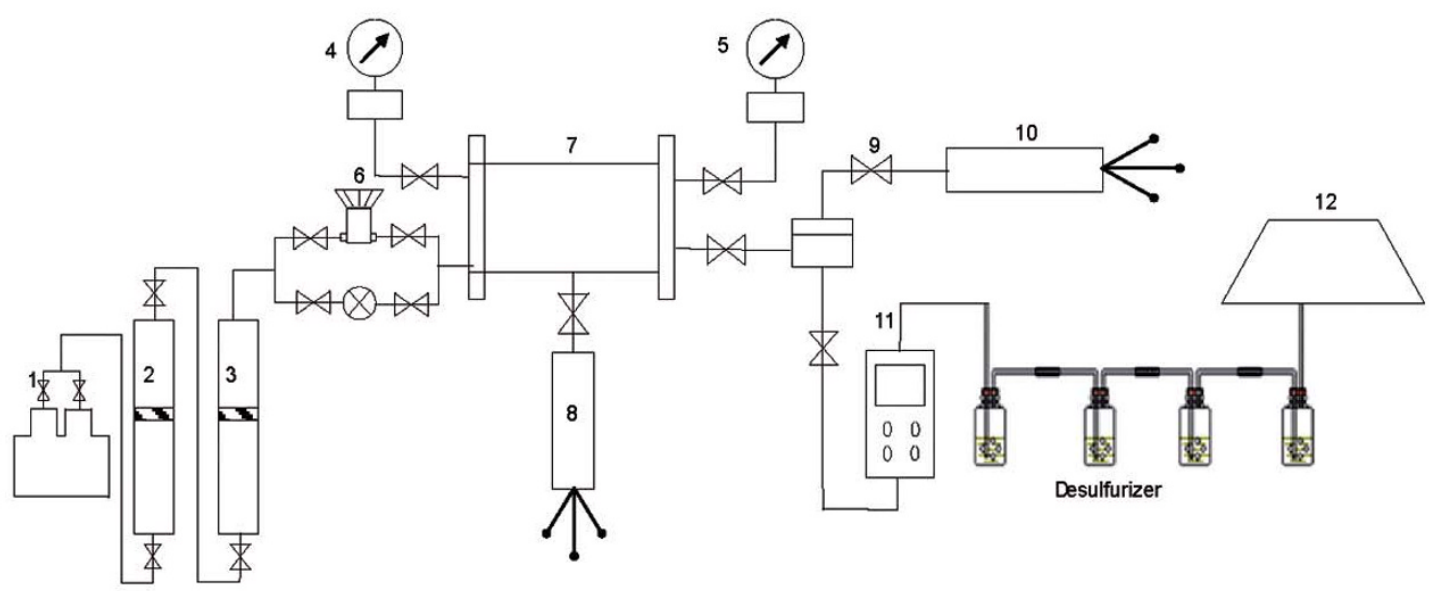

Fig. 1 Schematic diagram of experimental set up for sulfur deposition

1-Measuring pump; 2-Sample preparation; 3-Container; 4,5-Corrosion proof pressure gauge; 6-Voltage regulator; 7-Core holder; 8-Confining pressure pump; 9-Back pressure valve; 10-Back pressure pump; 11-Gas flow meter; 12-Ventilated fume hood

$$
叉, \bigotimes_{\text {Hand operated valve }}
$$

\subsection{Experimental results}

A set of dynamic flow experiments were carried out to investigate formation damage resulting from sulfur deposition. The core sample from LG2 well was used, the experimental temperature was $26^{\circ} \mathrm{C}$ and the initial pressure was $19 \mathrm{MPa}$. The confining pressure was kept as constant as $12 \mathrm{MPa}$. As the whole experimental process was in the stage of depletion, the displacement pressure decreased continuously from 19 to $10 \mathrm{MPa}$, and the depletion process lasted 15 days. Then the core was removed and dried, the core mass and core permeability were measured before and after the flow experiment. The result indicated that the core mass increased from 48.372 to $48.386 \mathrm{~g}$, while the core permeability reduced from 0.726 to $0.608 \mathrm{md}$, as shown in Table 2 . Then the core was analyzed by scanning electron microscope (SEM) and energy spectrum. The deposition pattern and micro-distribution of elemental sulfur was observed and the deposited elemental sulfur filmily distributed around the pore surface, as shown in Fig. 2.

Table 2 Variations in the core mass and core permeability before and after the flow experiment

\begin{tabular}{ccc}
\hline & $\begin{array}{c}\text { Core mass } \\
\mathrm{g}\end{array}$ & $\begin{array}{c}\text { Core permeability } \\
\mathrm{mD}\end{array}$ \\
Before the experiment & 48.372 & 0.726 \\
After the experiment & 48.386 & 0.608 \\
Increment & 0.014 & -0.118 \\
\hline Rate of change, \% & 0.029 & 16.253 \\
\hline
\end{tabular}

\section{एथ $\otimes$ Voltage regulator tube}

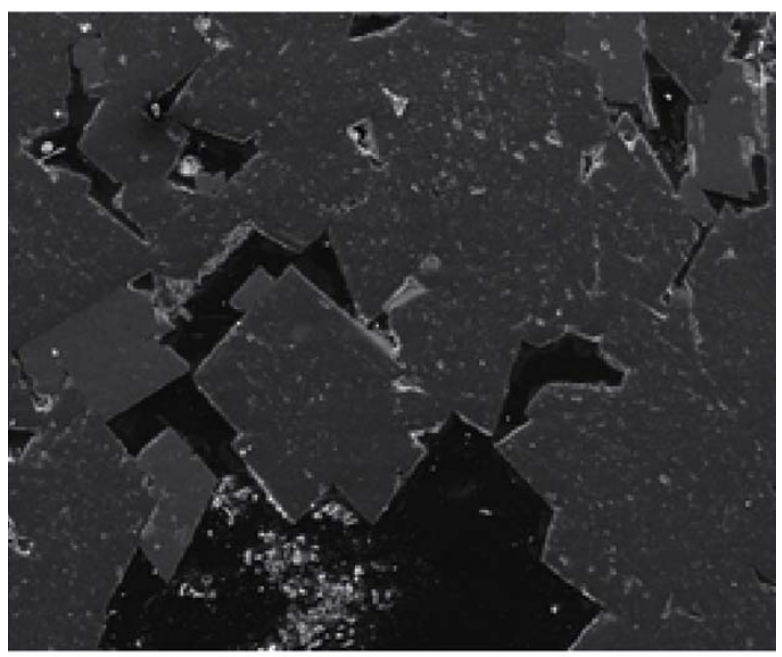

(a) Backscattered-electron image

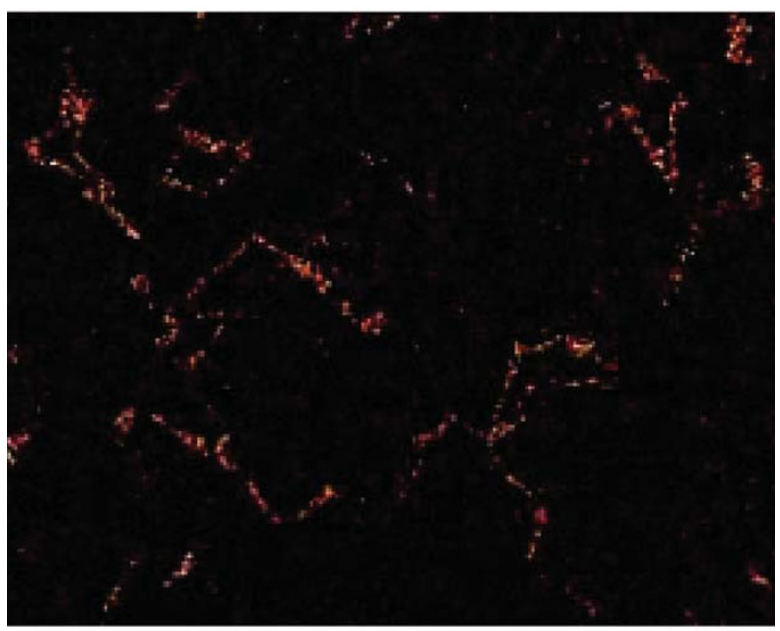

(b) S K-alpha X-ray map

Fig. 2 Distribution of sulfur in a polished section cut from the experimental core after sulfur deposition 


\section{Simulation investigation of sulfur deposition}

\subsection{Assumptions}

To simplify the coupled gas-liquid-solid flow mathematical model and be convenient to solve it, the following assumptions were made.

1) The temperature remains constant in the formation.

2) Fluid flow obeys Darcy's law relative to the sulfur solid phase flow.

3) Porosity and permeability are changed with pressure and sulfur deposition.

4) Media deformation is considered and the deformation is small.

5) The solubility of the sulfur in the gas phase was saturated at initial time.

\subsection{Differential equations}

The differential equations governing the flow of water, gas, and sulfur solid components in porous media can be written in the abbreviated form:

$$
\begin{aligned}
& \nabla\left(\frac{\rho_{\mathrm{g}} K}{\mu_{\mathrm{g}}} \nabla P\right)=\frac{\partial\left(\phi S_{\mathrm{g}} \rho_{\mathrm{g}}\right)}{\partial t}+\frac{q_{\mathrm{g}}}{V_{\mathrm{p}}} \\
& \nabla\left(\frac{K}{\mu_{\mathrm{g}}} \nabla P\right)+\nabla\left(u_{\mathrm{s}}\right)=\frac{\partial}{\partial t}\left(S_{\mathrm{g}} C_{\mathrm{s}}+C_{\mathrm{s}}^{\prime} S_{\mathrm{g}}+S_{\mathrm{s}}\right) \phi+\frac{q_{\mathrm{s}}}{V_{\mathrm{p}} \rho_{\mathrm{s}}}
\end{aligned}
$$

and

$$
\nabla\left[\frac{K \rho_{\mathrm{g}} Z_{\mathrm{g}}^{m}}{\mu_{\mathrm{g}}} \nabla P\right]=\frac{\partial\left[\phi\left(S_{\mathrm{g}} \rho_{\mathrm{g}} Z_{\mathrm{g}}^{m}\right)\right]}{\partial t}+\frac{q_{\mathrm{g}}}{V_{\mathrm{p}}} Z_{\mathrm{g}}^{m} \quad(m=1,2, \cdots)
$$

where $K$ is permeability, $10^{-3} \mu \mathrm{m}^{2} ; p$ is pressure, $\mathrm{MPa} ; \varphi$ is porosity; $\rho_{\mathrm{g}}$ is the density of gas, $\mathrm{g} / \mathrm{cm}^{3} ; \rho_{\mathrm{s}}$ is the density of sulfur, $\mathrm{g} / \mathrm{cm}^{3} ; S_{\mathrm{g}}$ is the gas saturation; $S_{\mathrm{s}}$ is the sulfur saturation; $u_{\mathrm{s}}$ is the migration velocity of sulfur particles, $\mathrm{cm} /$ $\mathrm{s} ; \mu_{\mathrm{g}}$ is the gas viscosity, $\mu \mathrm{Pa} \cdot \mathrm{s} ; t$ is time, $\mathrm{s} ; q_{\mathrm{g}}$ is source/sink term for gas, $\mathrm{m}^{3} / \mathrm{d} ; q_{\mathrm{s}}$ is source/sink term for elment sulfur, $\mathrm{m}^{3} / \mathrm{d} ; V_{\mathrm{p}}$ is the volume of per unit, $\mathrm{m}^{3} ; C_{\mathrm{s}}$ is the elemental sulfur solubility in gas mixture, $\mathrm{g} / \mathrm{m}^{3} ; C_{\mathrm{s}}^{\prime}$ is the suspended sulfur particle concentration in gas mixture, $\mathrm{g} / \mathrm{m}^{3} ; Z_{\mathrm{g}}{ }^{m}$ is the mole fraction of $m$ component in gas phase.

\subsection{Calculation of the separated-out mass of elemental sulfur}

A simple correlation developed by Chrastil (1982) for predicting the solubility of solids in a high pressure fluid was used to evaluate the desired solubility-pressure relationships:

$$
C_{\mathrm{r}}=\rho_{\mathrm{g}}^{\mathrm{k}} \exp \left(\frac{A}{T}+B\right)
$$

The above equation has been used extensively to correlate solubility data for the design of supercritical fluid extraction processes (Sung and Johnson, 1989). The separated-out mass of elemental sulfur was calculated in light of the above equation.

It is assumed that the solubility of elemental sulfur in the gas is $C_{\mathrm{r} 1}$, and the density is $\rho_{\mathrm{g} 1}$ at the time of $t_{1}$ in a cell, while the solubility of elemental sulfur is $C_{\mathrm{r} 2}$ and the density is $\rho_{\mathrm{g} 2}$ at the time of $t_{2}$, and that the temperature does not change in the time interval between $t_{1}$ and $t_{2}$, the separated-out mass of elemental sulfur for a unit of volume $V$ be expressed as follows:

$$
\Delta M_{\mathrm{rs}}=\Delta x \Delta y \Delta z \phi S_{\mathrm{g}}\left(C_{\mathrm{r} 1}-C_{\mathrm{r} 2}\right)
$$

Substituting Eq. (4) into Eq. (5) gives the model to calculate separated-out mass of elemental sulfur:

$$
\Delta M_{\mathrm{rs}}=V \phi S_{\mathrm{g}}\left(\rho_{\mathrm{g} 1}^{k}-\rho_{\mathrm{g} 2}^{k}\right) \exp \left(\frac{A}{T}+B\right)
$$

\subsection{Calculation of migration velocity of sulfur particle in the gas mixture}

Neglecting the clashes that may happen among sulfur particles in the gas mixture, it can be assumed that sulfur particles have the same velocity in the same cell. Thus, by means of the method of particle dynamics, the calculation of migration velocity of sulfur particle in gas mixture is as follows:

$$
u_{\mathrm{s}}=\sqrt{\frac{b}{a}}\left[\frac{1+\exp (4 t \sqrt{a b})}{1-\exp (4 t \sqrt{a b})}+2 \sqrt{\left[\frac{1+\exp (4 t \sqrt{a b})}{1-\exp (4 t \sqrt{a b})}\right]^{2}-1}\right]
$$

with

$$
\begin{gathered}
a=\frac{\rho C_{\mathrm{D}} \pi r_{\mathrm{p}}^{2}}{2 m_{\mathrm{p}}} \\
b=\frac{V_{\mathrm{p}}}{m_{\mathrm{p}}} \frac{\partial p}{\partial x}
\end{gathered}
$$

where $\rho$ is the density of the mixture of gas phase and solid phase, $\mathrm{kg} / \mathrm{m}^{3} ; C_{\mathrm{D}}$ is the resistance coefficient; $r_{\mathrm{p}}$ is the particle radius, $\mathrm{m} ; V_{\mathrm{p}}$ is the particle volume, $\mathrm{m}^{3} ; m_{\mathrm{p}}$ is the particle mass, $\mathrm{kg}$.

\subsection{Calculation of deposition velocity of sulfur particle in gas mixture}

The resistances to gas and solid in the course of migration in the conduit are related to gas/solid ratio, gas velocity, velocity of suspended particles, diameter and shape of conduit, and gas velocity, so the energy loss caused by the resistances can be categorized into two types: energy loss caused by friction between gas and conduit wall, and energy loss caused by clash and friction both between particles and between particle and gas.

By solving a series of equations (Du et al, 2006), the following equation can be obtained:

$$
u_{\mathrm{g}}=\sqrt[3]{\frac{m_{\mathrm{p}} D u_{\mathrm{mg}}}{\phi\left(\lambda_{\mathrm{g}}+\lambda_{\mathrm{m}} m_{\mathrm{p}} \phi\right)}}
$$


where $D$ is the pipe diameter, $\mathrm{m} ; u_{\mathrm{mg}}$ is the velocity of gas phase and solid phase, $\mathrm{m} / \mathrm{s} ; \lambda_{\mathrm{g}}$ is the gas friction coefficient; $\lambda_{\mathrm{m}}$ is the solid friction coefficient; $\varphi$ is the porosity.

Eq. (8) is the critical gas flow velocity with suspended particles. If gas flow velocity is less than the critical gas flow velocity with suspended particles, suspended particles will be deposited.

\subsection{Sulfur adsorption model}

Adsorption of sulfur can be considered to take place from the monomer phase. Sulfur adsorption formula can be expressed as:

$$
n_{\mathrm{s}}^{\prime}=\frac{m_{\mathrm{s}} x_{\mathrm{s}} S}{S x_{\mathrm{s}}+\left(m_{\mathrm{s}} / m_{\mathrm{g}}\right) x_{\mathrm{g}}}
$$

where $n_{\mathrm{s}}^{\prime}$ is the solid adsorption quantity; $m_{\mathrm{s}}$ is the mass number of sulfur particle per unit mass in absorption layer; $x_{\mathrm{s}}$ is the mass fraction of solid phase in mixture in continuous phase; $S$ is the selectivity factor; $m_{\mathrm{g}}$ is the mass number of gas per unit mass in absorption layer; $x_{\mathrm{g}}$ is the mass fraction of gas phase in mixture in continuous phase.

\subsection{Formation damage model}

Sulfur deposition can induce a reduction in formation porosity and permeability and the depositional rate is accelerated rapidly as the rock permeability decreases.

It is assumed that the volume of deposited-sulfur is invariable while the pressure is changing. So the porosity damage model is as follows:

$$
\phi=\phi_{0}-\Delta \phi=\phi_{0}-\frac{V_{\mathrm{s}}}{V} \times 100 \%
$$

where $V_{\mathrm{s}}$ is the volume of deposited elemental sulfur; $\varphi_{0}$ is the initial porosity; $V$ is the pore volume.

The permeability damage model presented here is based on the theory developed by Gruesbeck and Collins (Hyne, 1968) who originally developed the theory to describe entrainment and deposition of fines in porous media. They suggested hypothetical division of the porous medium into pluggable and nonpluggable pathways. This involves the representation of the porous medium into two continuous branches formed in such a way that one is of smaller pores that can be eventually plugged completely. On the other hand, the nonpluggable pathways cannot be completely plugged because as the pore throat diameter is reduced due to solid deposition, the local speed becomes high enough to entrain deposits out of the pore spaces. Thus, permeability damage model is as follows:

$$
K=f_{\mathrm{p}} K_{\mathrm{p} 0} \exp \left(-\alpha \varepsilon_{\mathrm{p}}^{n 1}\right)+f_{\mathrm{np}} K_{\mathrm{np} 0}\left(1+\beta \varepsilon_{\mathrm{p}}\right)
$$

where $K$ is the permeability, $10^{-3} \mu \mathrm{m}^{2} ; f_{\mathrm{p}}$ is the fraction of pore space containing pluggable pathways; $f_{\mathrm{np}}$ is the fraction of pore space containing nonpluggable pathways; $\alpha$ and $\beta$ are phenomenological constants to be specified; $\varepsilon$ is the volume of fines deposited per unit initial pore volume, $\mathrm{cm}^{3} / \mathrm{cm}^{3}$; subscript $\mathrm{p}$ represents pluggable pathways; $K_{\mathrm{p} 0}$ is the initial permeability of pore space containing pluggable pathways; $K_{\text {np } 0}$ is the initial permeability of pore space containing nonpluggable pathways.

\section{Computer model}

Based on the above mentioned mathematical models, a preliminary three-dimensional, multi-component, three-phase (gas-water-solid) flow numerical reservoir simulator was developed. A detail numerical model was listed in Appendix A. The program code was written in Visual Basic and the computing flow diagram is presented in Fig. 3.

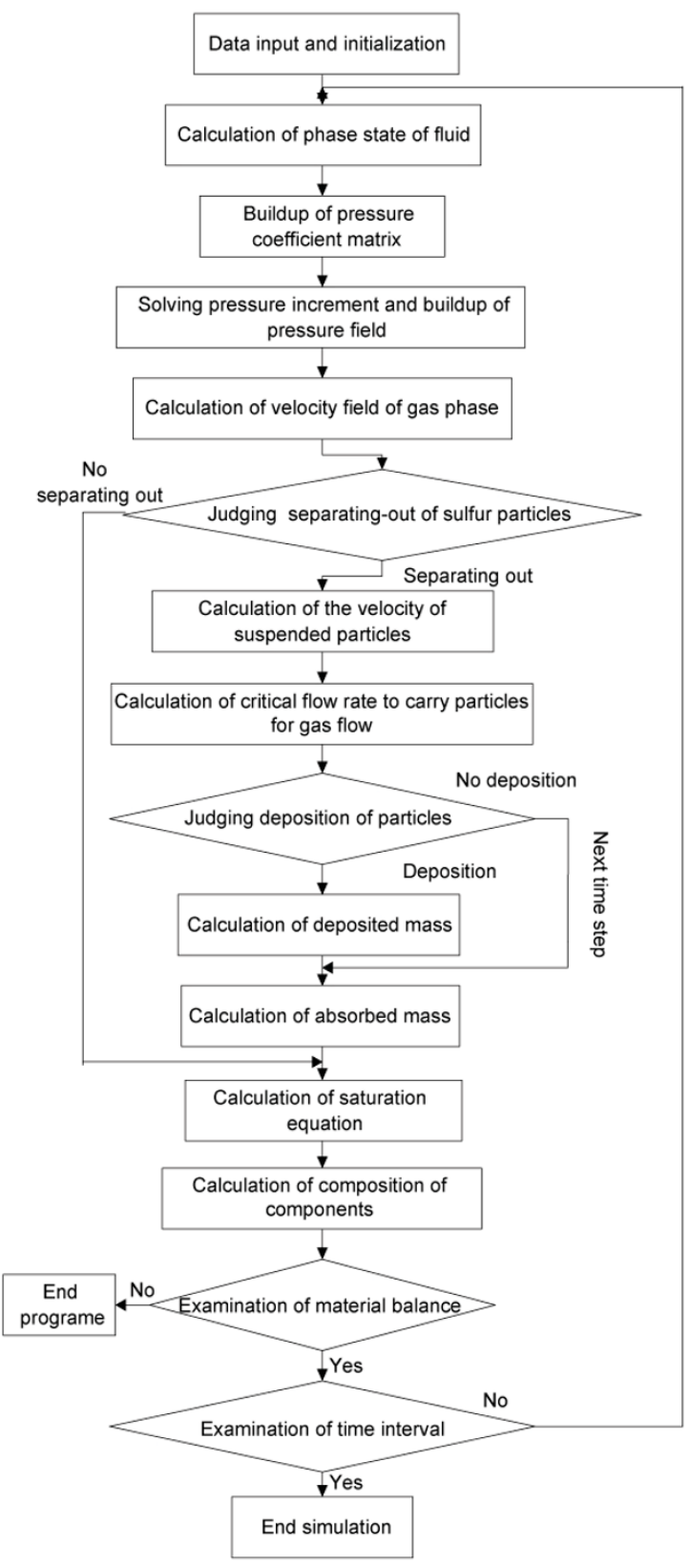

Fig. 3 The flow chart of simulator calculation

\subsection{Evaluation of the mass of the elemental sulfur}

Under reservoir conditions, the solubility of sulfur in the gas phase was $0.94 \mathrm{~g} / \mathrm{m}^{3}$, and the initial sulfur content 
in gas phase was $0.75 \mathrm{~g} / \mathrm{m}^{3}$. Therefore, sulfur in gas phase was undersaturated under reservoir conditions. With gas production, reduction in pressure and temperature will cause sulfur to deposit. Solubility of sulfur in the gas phase reaches the critical saturation state when the reservoir pressure decreases to $17.4 \mathrm{MPa}$, as shown in Fig. 4. Gas volume in the core under reservoir pressure of $17.4 \mathrm{MPa}$ was 0.02203 $\mathrm{m}^{3}$ under reservoir conditions, while gas volume in the core under reservoir pressure of $10 \mathrm{MPa}$ was $0.01969 \mathrm{~m}^{3}$ under reservoir conditions.

In light of Eq. (5) or Eq. (6), the mass of elemental sulfur deposited in the dynamic flow experiment was evaluated to be $0.0143 \mathrm{~g}$. The values of deposited sulfur predicted by the model were in good accordance with experimental results.

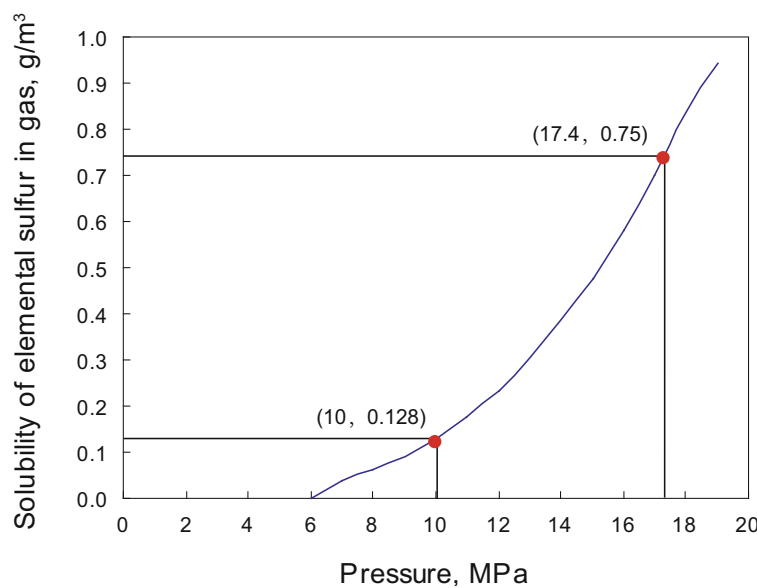

Fig. 4 The solubility of sulfur in the gas phase with pressure at the reservoir temperature

\subsection{Simulation of sulfur deposition}

The gas-liquid-solid coupling model presented by Du et al (2006) was used to evaluate the influences of sulfur deposition on stable production time, cumulative production, and reservoir pressure. The effect of production rate on sulfur deposition was also investigated. The parameters of the test cases are shown in Table 3 and Table 4.

Table 3 Reservoir properties

\begin{tabular}{cc}
\hline Parameters & Value \\
\hline Reservoir temperature, C & 26 \\
Initial pressure, $\mathrm{MPa}$ & 19 \\
Initial sulfur content, $\mathrm{g} / \mathrm{m}^{3}$ & 0.75 \\
Initial sulfur saturation, $\%$ & 0 \\
Pay thickness, $\mathrm{m}$ & 26 \\
$\quad$ Porosity & 0.032 \\
Permeability, $\times 10^{-3} \mu \mathrm{m}^{2}$ & 0.726 \\
Grid dimension & $11 \times 11 \times 1$ \\
\hline
\end{tabular}

Table 4 Reservoir fluid composition

\begin{tabular}{cc}
\hline Component & Mole fraction \\
\hline $\mathrm{N}_{2}$ & 0.0100 \\
$\mathrm{H}_{2} \mathrm{~S}$ & 0.1900 \\
$\mathrm{CO}_{2}$ & 0.0290 \\
$\mathrm{C}_{1}$ & 0.7600 \\
$\mathrm{C}_{2}$ & 0.0060 \\
$\mathrm{C}_{3+}$ & 0.0050 \\
\hline
\end{tabular}

The effects of sulfur deposition on the stable production time, cumulative production, and reservoir pressure were simulated, as shown in Figs. 5, 6, and 7. These results indicated that the stable production time would be shortened and the gas production rate would be decreased once sulfur deposited in the formation. Sulfur deposition could further cause a decrease in reservoir pressure. The effect of production rate on sulfur deposition was investigated, as shown in Fig. 8. The increase in deposited sulfur at high flow rates may be attributed to a greater pressure drop than at low gas flow rates. Sulfur deposition was not made worse by controlling

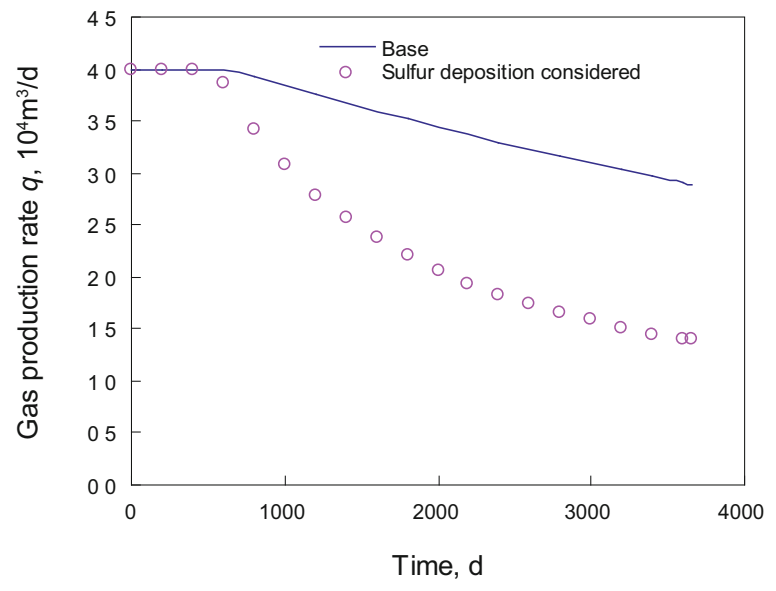

Fig. 5 The effect of sulfur deposition on gas production rate

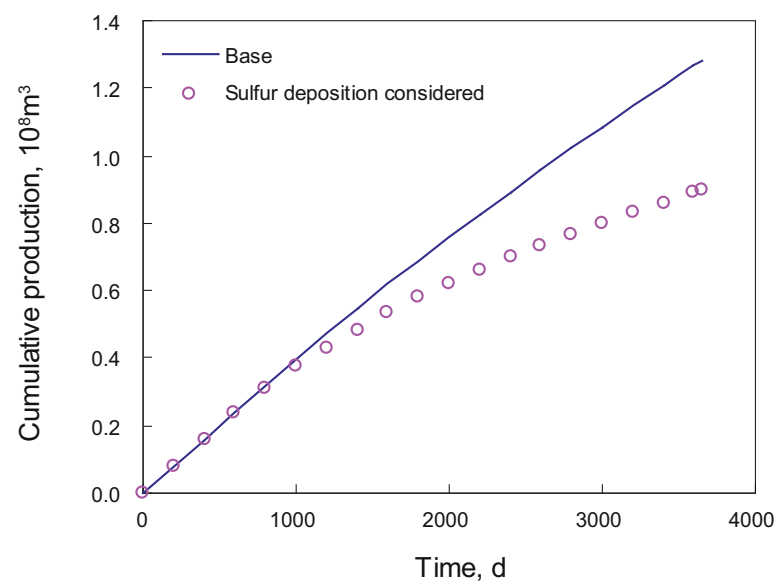

Fig. 6 The effect of sulfur deposition on cumulative production of gas 


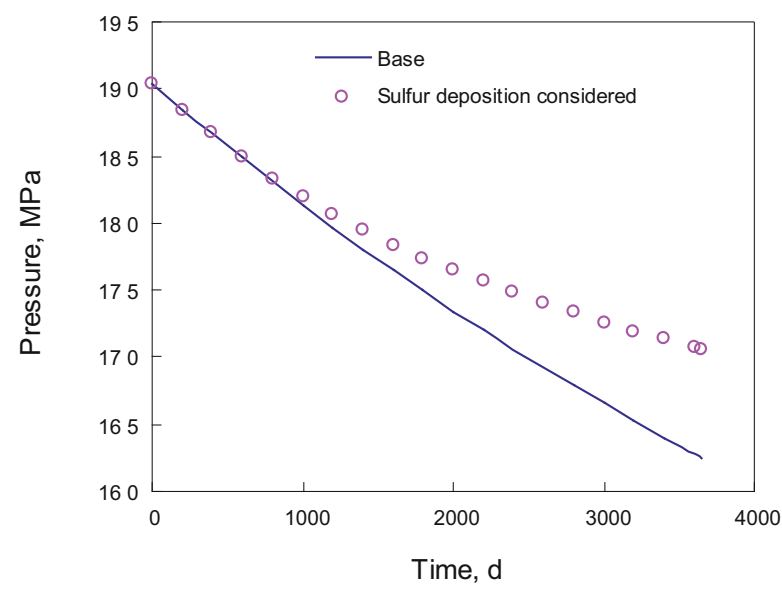

Fig. 7 The effect of sulfur deposition on reservoir pressure

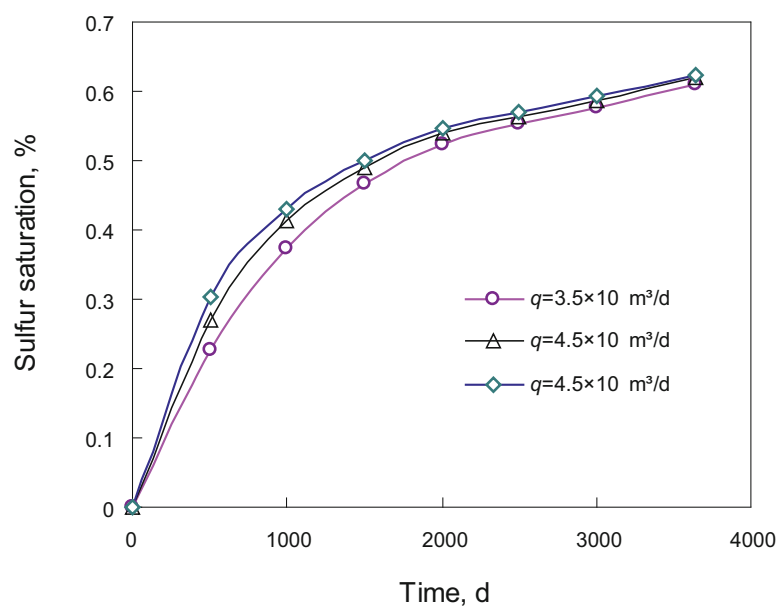

Fig. 8 The effect of the gas flow rate on sulfur deposition

the production flow rate of gas. The work suggests sulfur deposition should be considered to correctly predict production performance during the development of sour gas reservoirs.

\section{Conclusions}

1) Dynamic flow experiments were carried out to investigate formation damage resulting from sulfur deposition on the basis of improved experimental method. Experimental results indicated that the core mass increased from 48.372 to $48.386 \mathrm{~g}$, while the core permeability reduced from $0.726 \mathrm{mD}$ before the experiment to $0.608 \mathrm{mD}$ afterwards.

2) A polished section from the core was examined by a scanning electron microscope equipped with an energy dispersive X-ray spectrometer. The deposition pattern and micro-distribution of elemental sulfur was observed and the deposited elemental sulfur was distributed as a film on the pore surfaces.

3) A preliminary three-dimensional, multi-component model was developed to evaluate the influences of sulfur deposition on production performance and the effect of production rate on sulfur deposition was also investigated. Simulation results indicated that the stable production time would be shortened and the gas production rate would be decreased once sulfur deposited in the formation.

4) The increase in deposited sulfur at high flow rates may be attributed to a bigger pressure drop than that at low gas flow rates. Gas production rate has a severe effect on sulfur saturation in the grid of producing wells located in sour gas reservoirs.

5) The work suggests sulfur deposition should be considered to correctly predict production performance and gas production rate should be optimized in order to control or retard sulfur deposition during the development of sour gas reservoir.

\section{Acknowledgments}

This work was supported by the National High Technology Research and Development Program of China (863 Program) (No. 2007AA06Z209) and the National Natural Science Foundation of China (No. 50974104, 50774062 and 50474039). Technical support of State Key Laboratory of Oil and Gas Reservoir Geology and Exploitation we are working is also gratefully acknowledged.

\section{References}

Abou-Kassem J H. Experimental and numerical modeling of sulfur plugging in carbonate reservoirs. Journal of Petroleum Science and Engineering. 2000. 26(1-4): 91-103

Aitani A M. Sour natural gas drying. Hydrocarbon Processing. 1993. 72(4): 67-73

Al-Awadhy F, Kocabas I, Abou-Kassem J H, et al. Experimental and numerical modeling of sulfur plugging in carbonate oil reservoir. Abu Dhabi International Petroleum Exhibition and Conference held in Abu Dhabi, U.A.E, November 11-14, 1998 (SPE paper 49498)

Beskov V S, Kandybin Al and Furmer Y V. Modeling of the process of the removal of sulfur on zinc oxide absorbents in ammonia production. Russ. Chem. Ind. 1989. 21(3): 87-93

Brunner E, Place Jr M C and Woll W H. Sulfur solubility in sour gas. JPT. 1988. 40(12): 1587-1592

Brunner E and Woll W H. Solubility of sulfur in hydrogen sulfide and sour gases. Soc. Pet. Eng. J. 1980. 20(5): 377-384

Chrastil J. Solubility of solids and liquids in supercritical gas. J. Phys. Chem. 1982. 86: 3016

Du Z M, Guo X, Zhang Y, et al. Gas-liquid-solid coupled flow modelling in fractured carbonate gas reservoir with high $\mathrm{H}_{2} \mathrm{~S}$-content. SPE the First International Oil Conference and Exhibition held in Cancun, Mexico, 31 August-2 September, 2006 (SPE paper 103946)

Flowers D G. Use of permeation devices in the analysis of sulfur gases by gas chromatography. Ind. Eng. Chem. Res. 1990. 29(7): 15651568

Gasiorek J. Microbial removal of sulfur dioxide from a gas stream. Fuel Processing Technolgy. 1994. 40(2-3): 129-138

Hyne J B. Study aids prediction of sulfur deposition in sour-gas wells. Oil and Gas Journal.1968. 25(11): 107-113

Hyne J B. Controlling sulfur deposition in sour gas wells. World Oil. 1983. 197(2): 35-39

Hyne J B and Derdall G. Sulfur deposition in reservoirs and production equipment: sources and solutions. Annual Gas Conditioning Conference, University of Oklahoma, Norman, Oklahoma, March 3-5, 1980

Kuo C H and Colsmann P J. Theoretical study of fluid flow accompanied by solid precipitation in porous media. AICHE Journal. 1966. 12(5): 995-998

Roberts B E. The effect of sulfur deposition on gaswell inflow 
performance. SPE Reservoir Engineering. 1997. 12(2): 118-123 (SPE paper 36707 (1996))

Ruitenberg R, Dijkman H and Buisman C J N. Biologically removing sulfur from dilate gas flows. J. Miner. Met. Mater. 1999. 51(5): $45-49$

Shedid S A and Zekri A A. An experimental approach of elemental sulfur $\mathrm{d}$ position $\mathrm{n}$ carbonate oi reservoirs. Journal of Petroleum Science and Technology 2002. 20(5 6): 507-523

\section{Appendix A}

\section{Finite-difference equation}

The differential equations governing the flow of gas, sulfur and non-sulfur components in a porous medium can be written in the abbreviated form:

$$
\left\{\begin{array}{l}
\nabla\left(\frac{\rho_{\mathrm{g}} K}{\mu_{\mathrm{g}}} \nabla P\right)=\frac{\partial\left(\varphi S_{\mathrm{g}} \rho_{\mathrm{g}}\right)}{\partial t}+\frac{q_{\mathrm{g}}}{V_{\mathrm{p}}} \\
\nabla\left(\frac{K}{\mu_{\mathrm{g}}} \nabla P\right)+\nabla\left(u_{\mathrm{s}}\right)=\frac{\partial}{\partial t}\left(S_{\mathrm{g}} C_{\mathrm{s}}+C_{\mathrm{s}}^{\prime} S_{\mathrm{g}}+S_{\mathrm{s}}\right) \varphi+\frac{q_{\mathrm{s}}}{V_{\mathrm{p}} \rho_{\mathrm{s}}} \\
\nabla\left(\frac{K \rho_{\mathrm{g}} Z_{\mathrm{g}}^{m}}{\mu_{\mathrm{g}}} \nabla P\right)=\frac{\partial\left[\varphi\left(S_{\mathrm{g}} \rho_{\mathrm{g}} Z_{\mathrm{g}}^{m}\right)\right]}{\partial t}+\frac{q_{\mathrm{g}}}{V_{\mathrm{p}}} Z_{\mathrm{g}}^{m} \quad(m=1,2,3, \cdots)
\end{array}\right.
$$

The numerical solution of partial differential equations by finite differences involves replacing the partial derivatives by finite difference quotients. Then, instead of obtaining a continuous solution, an approximate solution was obtained at a discrete set of grid blocks or points at discrete times.

In expanded form, the differential equations ( Eq. (A-1)) are For gas

$$
\begin{aligned}
& \frac{1}{\Delta x_{i}}\left[\left(\frac{\rho_{\mathrm{g}} K}{\mu_{\mathrm{g}}}\right)_{i+\frac{1}{2}} \frac{P_{i+1}^{n+1}-P_{i}^{n+1}}{\Delta x_{i+\frac{1}{2}}}+\left(\frac{\rho_{\mathrm{g}} K}{\mu_{\mathrm{g}}}\right)_{i-\frac{1}{2}} \frac{P_{i-1}^{n+1}-P_{i}^{n+1}}{\Delta x_{i-\frac{1}{2}}}\right]+\frac{1}{\Delta y_{j}}\left[\left(\frac{\rho_{\mathrm{g}} K}{\mu_{\mathrm{g}}}\right)_{j+\frac{1}{2}} \frac{P_{j+1}^{n+1}-P_{j}^{n+1}}{\Delta y_{j+\frac{1}{2}}}+\left(\frac{\rho_{\mathrm{g}} K}{\mu_{\mathrm{g}}}\right)_{j-\frac{1}{2}} \frac{P_{j-1}^{n+1}-P_{j}^{n+1}}{\Delta y_{j-\frac{1}{2}}}\right]+ \\
& \frac{1}{\Delta z_{k}}\left[\left(\frac{\rho_{\mathrm{g}} K}{\mu_{\mathrm{g}}}\right)_{k+\frac{1}{2}} \frac{P_{k+1}^{n+1}-P_{k}^{n+1}}{\Delta z_{k+\frac{1}{2}}}+\left(\frac{\rho_{\mathrm{g}} K}{\mu_{\mathrm{g}}}\right)_{k-\frac{1}{2}} \frac{P_{k-1}^{n+1}-P_{k}^{n+1}}{\Delta z_{k-\frac{1}{2}}}\right]=\frac{\left(\varphi S_{\mathrm{g}} \rho_{\mathrm{g}}\right)^{n+1}-\left(\varphi S_{\mathrm{g}} \rho_{\mathrm{g}}\right)^{n}}{\Delta t}+\frac{q_{\mathrm{g}}}{V_{\mathrm{p}}}
\end{aligned}
$$

For sulfur component

$$
\begin{aligned}
& \frac{1}{\Delta x_{i}}\left[\left(\frac{K}{\mu_{\mathrm{g}}}\right)_{i+\frac{1}{2}} \frac{P_{i+1}^{n+1}-P_{i}^{n+1}}{\Delta x_{i+\frac{1}{2}}}+\left(\frac{K}{\mu_{\mathrm{g}}}\right)_{i-\frac{1}{2}} \frac{P_{i-1}^{n+1}-P_{i}^{n+1}}{\Delta x_{i-\frac{1}{2}}}\right]+\frac{1}{\Delta y_{j}}\left[\left(\frac{K}{\mu_{\mathrm{g}}}\right)_{j+\frac{1}{2}} \frac{P_{j+1}^{n+1}-P_{j}^{n+1}}{\Delta y_{j+\frac{1}{2}}}+\left(\frac{K}{\mu_{\mathrm{g}}}\right)_{j-\frac{1}{2}} \frac{P_{j-1}^{n+1}-P_{j}^{n+1}}{\Delta y_{j-\frac{1}{2}}}\right]+ \\
& \frac{1}{\Delta z_{k}}\left[\left(\frac{K}{\mu_{\mathrm{g}}}\right)_{k+\frac{1}{2}} \frac{P_{k+1}^{n+1}-P_{k}^{n+1}}{\Delta z}+\left(\frac{K}{\mu_{\mathrm{g}}}\right)_{k-\frac{1}{2}} \frac{P_{k-1}^{n+1}-P_{k}^{n+1}}{\Delta z_{k-\frac{1}{2}}}\right]+\frac{1}{\Delta x_{i}}\left[u_{\mathrm{s}, i+1}^{n+1}-u_{\mathrm{s}, i}^{n+1}\right]+\frac{1}{\Delta y_{j}}\left[u_{\mathrm{s}, j+1}^{n+1}-u_{\mathrm{s}, j}^{n+1}\right]+\frac{1}{\Delta z_{i}}\left[u_{\mathrm{s}, k+1}^{n+1}-u_{\mathrm{s}, k}^{n+1}\right] \\
& =\frac{\left[\left(S_{\mathrm{g}} C_{\mathrm{s}}+C_{\mathrm{s}}^{\prime} S_{\mathrm{g}}+S_{\mathrm{s}}\right) \varphi\right]^{n+1}-\left[\left(S_{\mathrm{g}} C_{\mathrm{s}}+C_{\mathrm{s}}^{\prime} S_{\mathrm{g}}+S_{\mathrm{s}}\right) \varphi\right]^{n}}{\Delta t}+\frac{q_{\mathrm{s}}}{V_{\mathrm{p}} \rho_{\mathrm{s}}}
\end{aligned}
$$

For non-sulfur components

$$
\begin{aligned}
& \left.\frac{1}{\Delta x_{i}}\left[\left(\frac{\rho_{\mathrm{g}} K Z_{\mathrm{g}}^{m}}{\mu_{\mathrm{g}}}\right)_{i+\frac{1}{2}} \frac{P_{i+1}^{n+1}-P_{i}^{n+1}}{\Delta x_{i+\frac{1}{2}}}+\left(\frac{\rho_{\mathrm{g}} K Z_{\mathrm{g}}^{m}}{\mu_{\mathrm{g}}}\right)_{i-\frac{1}{2}} \frac{P_{i-1}^{n+1}-P_{i}^{n+1}}{\Delta x_{i-\frac{1}{2}}}\right]+\frac{1}{\Delta y_{j}}\left[\left(\frac{\rho_{\mathrm{g}} K Z_{g}^{m}}{\mu_{g}}\right)_{j+\frac{1}{2}} \frac{P_{j+1}^{n+1}-P_{j}^{n+1}}{\Delta y_{j+\frac{1}{2}}}+\left(\frac{\rho_{\mathrm{g}} K Z_{g}^{m}}{\mu_{g}}\right)_{j-\frac{1}{2}} \frac{P_{j-1}^{n+1}-P_{j}^{n+1}}{\Delta y}\right]_{j-\frac{1}{2}}\right] \\
& \frac{1}{\Delta z_{k}}\left[\left(\frac{\rho_{\mathrm{g}} K Z_{\mathrm{g}}^{m}}{\mu_{\mathrm{g}}}\right)_{k+\frac{1}{2}} \frac{P_{k+1}^{n+1}-P_{k}^{n+1}}{\Delta z_{k+\frac{1}{2}}}+\left(\frac{\rho_{\mathrm{g}} K Z_{\mathrm{g}}^{m}}{\mu_{\mathrm{g}}}\right)_{k-\frac{1}{2}} \frac{P_{k-1}^{n+1}-P_{k}^{n+1}}{\Delta z_{k-\frac{1}{2}}}\right]=\frac{\left(\varphi S_{\mathrm{g}} \rho_{\mathrm{g}} Z_{\mathrm{g}}^{m}\right)^{n+1}-\left(\varphi S_{\mathrm{g}} \rho_{\mathrm{g}} Z_{\mathrm{g}}^{m}\right)^{n}}{\Delta t}+\frac{q_{\mathrm{g}}}{V_{\mathrm{p}}} Z_{\mathrm{g}}^{m}
\end{aligned}
$$


By multiplying $\Delta x_{i} \Delta y_{j} \Delta z_{k}$, Eqs. (A-2), (A-3) and (A-4) can be written as

$$
\begin{aligned}
& \frac{\Delta y_{j} \Delta z_{k}}{\Delta x_{i+\frac{1}{2}}}\left(\frac{\rho_{\mathrm{g}} K}{\mu_{\mathrm{g}}}\right)_{i+\frac{1}{2}}\left(P_{i+1}^{n+1}-P_{i}^{n+1}\right)+\frac{\Delta y_{j} \Delta z_{k}}{\Delta x_{i-\frac{1}{2}}}\left(\frac{\rho_{\mathrm{g}} K}{\mu_{\mathrm{g}}}\right)_{i-\frac{1}{2}}\left(P_{i-1}^{n+1}-P_{i}^{n+1}\right)+\frac{\Delta x_{i} \Delta z_{k}}{\Delta y_{j+\frac{1}{2}}}\left(\frac{\rho_{\mathrm{g}} K}{\mu_{\mathrm{g}}}\right)_{j+\frac{1}{2}}\left(P_{j+1}^{n+1}-P_{j}^{n+1}\right)+ \\
& \frac{\Delta x_{i} \Delta z_{k}}{\Delta y_{j-\frac{1}{2}}}\left(\frac{\rho_{\mathrm{g}} K}{\mu_{\mathrm{g}}}\right)_{j-\frac{1}{2}}\left(P_{j-1}^{n+1}-P_{j}^{n+1}\right)+\frac{\Delta x_{i} \Delta y_{j}}{\Delta z_{k+\frac{1}{2}}}\left(\frac{\rho_{\mathrm{g}} K}{\mu_{\mathrm{g}}}\right)_{k+\frac{1}{2}}\left(P_{k+1}^{n+1}-P_{k}^{n+1}\right)+\frac{\Delta x_{i} \Delta y_{j}}{\Delta z_{k-\frac{1}{2}}}\left(\frac{\rho_{\mathrm{g}} K}{\mu_{\mathrm{g}}}\right)_{k-\frac{1}{2}}\left(P_{k-1}^{n+1}-P_{k}^{n+1}\right) \\
& =V_{\mathrm{p}} \frac{\left(\varphi S_{\mathrm{g}} \rho_{\mathrm{g}}\right)^{n+1}-\left(\varphi S_{\mathrm{g}} \rho_{\mathrm{g}}\right)^{n}}{\Delta t}+q_{\mathrm{g}} \\
& \frac{\Delta y_{j} \Delta z_{k}}{\Delta x_{i+\frac{1}{2}}}\left(\frac{K}{\mu_{\mathrm{g}}}\right)_{i+\frac{1}{2}}\left(P_{i+1}^{n+1}-P_{i}^{n+1}\right)+\frac{\Delta y_{j} \Delta z_{k}}{\Delta x_{i-\frac{1}{2}}}\left(\frac{K}{\mu_{\mathrm{g}}}\right)_{i-\frac{1}{2}}\left(P_{i-1}^{n+1}-P_{i}^{n+1}\right)+\frac{\Delta x_{i} \Delta y_{j}}{\Delta z_{k+\frac{1}{2}}}\left(\frac{K}{\mu_{\mathrm{g}}}\right)_{k+\frac{1}{2}}\left(P_{k+1}^{n+1}-P_{k}^{n+1}\right)+ \\
& \frac{\Delta x_{i} \Delta y_{j}}{\Delta z_{k-\frac{1}{2}}}\left(\frac{K}{\mu_{\mathrm{g}}}\right)_{k-\frac{1}{2}}\left(P_{k-1}^{n+1}-P_{k}^{n+1}\right)+\frac{\Delta x_{i} \Delta y_{j}}{\Delta z_{k+\frac{1}{2}}}\left(\frac{K}{\mu_{g}}\right)_{k+\frac{1}{2}}\left(P_{k+1}^{n+1}-P_{k}^{n+1}\right)+\frac{\Delta x_{i} \Delta y_{j}}{\Delta z_{k-\frac{1}{2}}}\left(\frac{K}{\mu_{g}}\right)_{k-\frac{1}{2}}\left(P_{k-1}^{n+1}-P_{k}^{n+1}\right)+ \\
& \Delta y_{j} \Delta z_{k}\left[u_{\mathrm{s}, i+1}^{n+1}-u_{\mathrm{s}, i}^{n+1}\right]+\Delta x_{i} \Delta z_{k}\left[u_{\mathrm{s}, j+1}^{n+1}-u_{s, j}^{n+1}\right]+\Delta x_{i} \Delta y_{j}\left[u_{\mathrm{s}, k+1}^{n+1}-u_{\mathrm{s}, k}^{n+1}\right] \\
& =V_{\mathrm{p}} \frac{\left[\left(S_{\mathrm{g}} C_{\mathrm{s}}+C_{\mathrm{s}}^{\prime} S_{\mathrm{g}}+S_{\mathrm{s}}\right) \varphi\right]^{n+1}-\left[\left(S_{\mathrm{g}} C_{\mathrm{s}}+C_{\mathrm{s}}^{\prime} S_{\mathrm{g}}+S_{\mathrm{s}}\right) \varphi\right]^{n}}{\Delta t}+\frac{q_{\mathrm{s}}}{\rho_{\mathrm{s}}} \\
& \frac{\Delta y_{j} \Delta z_{k}}{\Delta x_{i+\frac{1}{2}}}\left(\frac{\rho_{\mathrm{g}} K Z_{\mathrm{g}}^{m}}{\mu_{\mathrm{g}}}\right)_{i+\frac{1}{2}}\left(P_{i+1}^{n+1}-P_{i}^{n+1}\right)+\frac{\Delta y_{j} \Delta z_{k}}{\Delta x_{i-\frac{1}{2}}}\left(\frac{\rho_{\mathrm{g}} K Z_{\mathrm{g}}^{m}}{\mu_{\mathrm{g}}}\right)_{i-\frac{1}{2}}\left(P_{i-1}^{n+1}-P_{i}^{n+1}\right)+\frac{\Delta x_{i} \Delta z_{k}}{\Delta y_{j+\frac{1}{2}}}\left(\frac{\rho_{\mathrm{g}} K Z_{\mathrm{g}}^{m}}{\mu_{\mathrm{g}}}\right)_{j+\frac{1}{2}}\left(P_{j+1}^{n+1}-P_{j}^{n+1}\right)+ \\
& \frac{\Delta x_{i} \Delta z_{k}}{\Delta y_{j-\frac{1}{2}}}\left(\frac{\rho_{\mathrm{g}} K Z_{\mathrm{g}}^{m}}{\mu_{\mathrm{g}}}\right)_{j-\frac{1}{2}}\left(P_{j-1}^{n+1}-P_{j}^{n+1}\right)+\frac{\Delta x_{i} \Delta y_{j}}{\Delta z_{k+\frac{1}{2}}}\left(\frac{\rho_{\mathrm{g}} K Z_{\mathrm{g}}^{m}}{\mu_{\mathrm{g}}}\right)_{k+\frac{1}{2}}\left(P_{k+1}^{n+1}-P_{k}^{n+1}\right)+\frac{\Delta x_{i} \Delta y_{j}}{\Delta z_{k-\frac{1}{2}}}\left(\frac{\rho_{\mathrm{g}} K Z_{\mathrm{g}}^{m}}{\mu_{\mathrm{g}}}\right)_{k-\frac{1}{2}}\left(P_{k-1}^{n+1}-P_{k}^{n+1}\right) \\
& =V_{\mathrm{p}} \frac{\left(\varphi S_{\mathrm{g}} \rho_{\mathrm{g}} Z_{\mathrm{g}}^{m}\right)^{n+1}-\left(\varphi S_{\mathrm{g}} \rho_{\mathrm{g}} Z_{\mathrm{g}}^{m}\right)^{n}}{\Delta t}+q_{\mathrm{g}} Z_{\mathrm{g}}^{m}
\end{aligned}
$$

Each of the transmissibility terms is divided into two parts. i.e, one is the geometric factor and the other is the fluidity coefficient.

Let

$$
\begin{aligned}
& F_{i \pm \frac{1}{2}}=\frac{\Delta y_{j} \Delta z_{k}}{\Delta x_{i \pm \frac{1}{2}}}, \quad F_{j \pm \frac{1}{2}}=\frac{\Delta x_{i} \Delta z_{k}}{\Delta y_{j \pm \frac{1}{2}}}, \quad F_{k \pm \frac{1}{2}}=\frac{\Delta x_{i} \Delta y_{j}}{\Delta z_{k \pm \frac{1}{2}}}, \quad \lambda_{\mathrm{g}}=\frac{K}{\mu_{\mathrm{g}}}, \\
& f_{i}=\Delta y_{j} \Delta z_{k}, \quad f_{j}=\Delta x_{i} \Delta z_{k}, \quad f_{k}=\Delta x_{i} \Delta y_{j}
\end{aligned}
$$

The final form of the difference equation for gas is

$$
\begin{aligned}
& F_{i+\frac{1}{2}}\left(\rho_{\mathrm{g}} \lambda_{\mathrm{g}}\right)_{i+\frac{1}{2}}\left(P_{i+1}^{n+1}-P_{i}^{n+1}\right)+F_{i-\frac{1}{2}}\left(\rho_{\mathrm{g}} \lambda_{\mathrm{g}}\right)_{i-\frac{1}{2}}\left(P_{i-1}^{n+1}-P_{i}^{n+1}\right)+F_{j+\frac{1}{2}}\left(\rho_{\mathrm{g}} \lambda_{\mathrm{g}}\right)_{j+\frac{1}{2}}\left(P_{j+1}^{n+1}-P_{j}^{n+1}\right)+ \\
& F_{j-\frac{1}{2}}\left(\rho_{\mathrm{g}} \lambda_{\mathrm{g}}\right)_{j-\frac{1}{2}}\left(P_{j-1}^{n+1}-P_{j}^{n+1}\right)+F_{k+\frac{1}{2}}\left(\rho_{\mathrm{g}} \lambda_{\mathrm{g}}\right)_{k+\frac{1}{2}}\left(P_{k+1}^{n+1}-P_{k}^{n+1}\right)+F_{k-\frac{1}{2}}\left(\rho_{\mathrm{g}} \lambda_{\mathrm{g}}\right)_{k-\frac{1}{2}}\left(P_{k-1}^{n+1}-P_{k}^{n+1}\right) \\
& =V_{\mathrm{p}} \frac{\left(\varphi S_{\mathrm{g}} \rho_{\mathrm{g}}\right)^{n+1}-\left(\varphi S_{\mathrm{g}} \rho_{\mathrm{g}}\right)^{n}}{\Delta t}+q_{\mathrm{g}}
\end{aligned}
$$


Similarly, the difference equation for elemental sulfur is

$$
\begin{aligned}
& F_{i+\frac{1}{2}}\left(\lambda_{\mathrm{g}}\right)_{i+\frac{1}{2}}\left(\left(P_{i+1}^{n+1}-P_{i}^{n+1}\right)\right)+F_{i-\frac{1}{2}}\left(\left(\lambda_{\mathrm{g}}\right)_{i-\frac{1}{2}}\left(\left(P_{i-1}^{n+1}-P_{i}^{n+1}\right)\right)+F_{j+\frac{1}{2}}\left(\left(\lambda_{\mathrm{g}}\right)\right)_{j+\frac{1}{2}}\left(\left(P_{j+1}^{n+1}-P_{j}^{n+1}\right)\right)+\right. \\
& F_{j-\frac{1}{2}}\left(\lambda_{\mathrm{g}}\right)_{j-\frac{1}{2}}\left(P_{j-1}^{n+1}-P_{j}^{n+1}\right)+F_{k+\frac{1}{2}}\left(\lambda_{\mathrm{g}}\right)_{k+\frac{1}{2}}\left(P_{k+1}^{n+1}-P_{k}^{n+1}\right)+F_{k-\frac{1}{2}}\left(\lambda_{\mathrm{g}}\right)_{k-\frac{1}{2}}\left(P_{k-1}^{n+1}-P_{k}^{n+1}\right)+ \\
& f_{i}\left(u_{\mathrm{s}, i+1}^{n+1}-u_{\mathrm{s}, i}^{n+1}\right)+f_{j}\left(u_{\mathrm{s}, j+1}^{n+1}-u_{\mathrm{s}, j}^{n+1}\right)+f_{k}\left(u_{\mathrm{s}, k+1}^{n+1}-u_{\mathrm{s}, k}^{n+1}\right) \\
& =V_{\mathrm{p}} \frac{\left[\left(S_{\mathrm{g}} C_{\mathrm{s}}+C_{\mathrm{s}}^{\prime} S_{\mathrm{g}}+S_{\mathrm{s}}\right) \varphi\right]^{n+1}-\left[\left(S_{\mathrm{g}} C_{\mathrm{s}}+C_{\mathrm{s}}^{\prime} S_{\mathrm{g}}+S_{\mathrm{s}}\right) \varphi\right]^{n}}{\Delta t}+\frac{q_{\mathrm{s}}}{\rho_{\mathrm{s}}}
\end{aligned}
$$

and the difference equation for non-sulfur components is:

$$
\begin{aligned}
& F_{i+\frac{1}{2}}\left(\lambda_{\mathrm{g}} \rho_{\mathrm{g}} Z_{\mathrm{g}}^{m}\right)_{i+\frac{1}{2}}\left(P_{i+1}^{n+1}-P_{i}^{n+1}\right)+F_{i-\frac{1}{2}}\left(\lambda_{\mathrm{g}} \rho_{\mathrm{g}} Z_{\mathrm{g}}^{m}\right)_{i-\frac{1}{2}}\left(P_{i-1}^{n+1}-P_{i}^{n+1}\right)+F_{j+\frac{1}{2}}\left(\lambda_{\mathrm{g}} \rho_{\mathrm{g}} Z_{\mathrm{g}}^{m}\right)_{j+\frac{1}{2}}\left(P_{j+1}^{n+1}-P_{j}^{n+1}\right)+ \\
& F_{j-\frac{1}{2}}\left(\left(\lambda_{\mathrm{g}} \rho_{\mathrm{g}} Z_{\mathrm{g}}^{m}\right)\right)_{j-\frac{1}{2}}\left(P_{j-1}^{n+1}-P_{j}^{n+1}\right)+F_{k+\frac{1}{2}}\left(\lambda_{\mathrm{g}} \rho_{\mathrm{g}} Z_{\mathrm{g}}^{m}\right)_{k+\frac{1}{2}}\left(P_{k+1}^{n+1}-P_{k}^{n+1}\right)+F_{k-\frac{1}{2}}\left(\lambda_{\mathrm{g}} \rho_{\mathrm{g}} Z_{\mathrm{g}}^{m}\right)_{k-\frac{1}{2}}\left(P_{k-1}^{n+1}-P_{k}^{n+1}\right) \\
& =V_{\mathrm{p}} \frac{\left(\varphi S_{\mathrm{g}} \rho_{\mathrm{g}} Z_{\mathrm{g}}^{m}\right)^{n+1}-\left(\varphi S_{\mathrm{g}} \rho_{\mathrm{g}} Z_{\mathrm{g}}^{m}\right)^{n}}{\Delta t}+q_{\mathrm{g}} Z_{\mathrm{g}}^{m}
\end{aligned}
$$

